\title{
Sepsis, septicaemia, sepsis syndrome, and septic shock: the correct definition and use
}

Sepsis is an old medical term which comes from the Greek 'sepsin' meaning 'to make putrid'. Although most authors agree that sepsis refers to the systemic response to infection caused by any class of microorganisms, ${ }^{1-3}$ a single comprehensive and correct definition of this widely used term is still lacking. Also, in some medical dictionaries sepsis is defined incorrectly. For example, Stedman's Medical Dictionary ${ }^{4}$ defines this term as the presence of various pus-forming and other pathogenic organisms, or their toxins, in the blood or tissues. This definition does not reflect the presence of illness in the host, and thus it is a more suitable definition of bacteraemia, viraemia, endotoxaemia, etc, where the presence of these microorganisms or their toxins in the bloodstream does not always necessitate the occurrence of illness. So, sepsis should be defined as the systemic inflammatory response to infection caused by any class of microorganisms with the invasion of these microorganisms or their toxins in the bloodstream causing illness. This illness is clinically manifested by fever or hypothermia, tachycardia, and tachypnea, and it may be associated with various degrees of injury to one or more major organs. Thus, sepsis may be graded as mild, moderate, or severe, according to the severity of the associated organ or organs, injury and failure. When sepsis causes shock, this situation is defined as septic shock, and there is a consensus regarding this definition.

Some authors have recently begun to use the terms 'sepsis syndrome', and 'systemic inflammatory response syndrome' to describe the presence of severe sepsis or septic shock..$^{3,5,6}$ I believe that the introduction of these terms is unnecessary since the term sepsis with its degrees (mild, moderate, and severe), and septic shock, as defined above are comprehensive and more correct and clear.

The term 'septicaemia' is frequently used in the medical literature and still appears in most medical journals, ${ }^{7-9}$ and in recent editions of medical textbooks, ${ }^{1}$ and of medical dictionaries. ${ }^{4}$ Septicaemia is defined as a systematic disease caused by the spread of microorganisms and their toxins via the circulating blood. ${ }^{4}$ However, the term sepsis, as defined above, describes more clearly this situation, thus the term 'septicaemia' is unnecessary. Furthermore, the suffix

1 Kasper DL, Zaleznik DF. Gas gangrene and other clostridial infections. In Isselbacher KJ, Braunwald E, Wilson JD, Martin JB, Fauci AS, Kasper DL, eds. Harrison's principles of internal medicine, 13th edn. Vol 1 . New York: McGraw-Hill, 1994; pp 636-40.

2 Vincent JL, Bihari D. Sepsis, severe sepsis or sepsis syndrome: need for clarification. Intensive Care Med 1991; 18: 255-7.

3 Bone RC. Why new definitions of sepsis and organ failure are needed. $A m \mathcal{F}$ Med 1992; 95: 348-50.

4 Hensyl WR. Sepsis. Stedman's Medical Dictionary, 26th edn. Baltimore: Williams \& Wilkins, 1995; p 1598.

5 Sibbald WJ, McCormack D, Marshall J, et al. Sepsis - clarity of existing terminology ... or more confusion? Crit Care Med 1991; 19: 996-8.

\begin{tabular}{|l|}
\hline Definitions \\
\hline - Sepsis: the systemic inflammatory response to infection \\
caused by any class of microorganisms with the invasion of \\
these microorganisms or their toxins in the bloodstream \\
causing illness \\
- Septicaemia: a systemic disease caused by the spread of \\
microorganisms and their toxins via the circulating blood \\
- Sepsis syndrome: the association of sepsis with altered organ \\
perfusion and/or altered organ function \\
- Septic shock: a severe form of sepsis causing inadequate \\
tissue perfusion and shock
\end{tabular}

'aemia' means blood, and when it is connected with another word it means that something is present in the circulating blood, ${ }^{4} \mathrm{eg}$, bacteraemia when bacteria are present in the bloodstream. Thus, the term 'septicaemia' means that sepsis is present in the circulating blood. However, as sepsis refers to the systemic response to infection caused by any class of microorganisms or their toxins, and not a particle which can circulate in the bloodstream, the term 'septicaemia' is meaningless, confusing, and unsuitable. Thus, I suggest that the term 'septicaemia' is no longer used in the medical literature.

In summary, the appropriate terms to use to describe an infection by any microorganism when the microorganism or its toxin is present in the bloodstream are bacteraemia, viraemia, fungaemia, endotoxaemia, etc. When the invasion of these microorganisms or their toxins into the bloodstream causes illness, this may be defined as mild, moderate, or severe sepsis, or septic shock, whichever is appropriate.

MAJED ODEH

Department of Internal Medicine B, Bnai Zion Medical Center, and Technion Faculty of Medicine, Israel Institute of Technology, Haifa, Israel

6 Casey LC, Balk RA, Bone RC. Plasma cytokine and endotoxin levels correlate with survival in patients with the sepsis syndrome. Ann Intern Med 1993; 119: 771-8.

7 Leclerc F, Delepoulle F, Diependaele JF, et al. Severity scores in meningococcal septicaemia and severe infectious purpura with shock. Intensive Care Med 1995; 21: 264-5.

8 Berger C, Uehlinger J, Ghelfi D, et al. Comparison of C-reactive protein and white blood cell count with differential in neonates at risk for septicaemia. Eur $\mathcal{F}$ Pediatr 1995; 154: 138-44.

9 Rantale A, Vaahtorantalehtonen H. Hematogenous endophthalmitis in patients with postoperative septicaemia. Clin Infect Dis 1995; 20: 472-3. 